\title{
Embedded Experiment Teaching Practice based on System Design
}

\author{
Tao Chi $^{1, \text { a }}$ and Lei Wang ${ }^{2}$ \\ ${ }^{1}$ Shanghai Ocean University, Shanghai, China \\ ${ }^{2}$ Shanghai Ocean University, Shanghai, China \\ atchi@shou.edu.cn
}

\begin{abstract}
Keywords: Embedded system experiment; whole English teaching; embedded system; project
\end{abstract} oriented; data acquisition system; the ability of solving problem.

\begin{abstract}
Embedded system experiment is an important experiment course of computer science and technology which need a wider range of knowledge, higher requirements for designers and wealth of practical experience. Most college students reflect the existing embedded experimental courses, which are boring and difficult to master the essence of embedded design - the system level design, even worse the society needs is out of line with the curriculum. In order to solve such contradiction between theory and practice of the embedded system, this paper uses the embedded teaching platform provided by the laboratory (mini2440) for the design of hardware and software, dividing a data acquisition system into several modules, and then analyzing each of the module.The embedded experiment based on system design will ensure that most of the students can master the design of embedded system. This kind of teaching method not only improves the ability of solving problems, but also stimulates students' interest in system level design. Practice has proved that the teaching reform of embedded system is in line with the international advanced technology, and the teaching core is put into the practical application.
\end{abstract}

\section{Introduction}

In recent years, the embedded system has been rapidly developed from the SCM to the DSP \& ARM, infiltrated into mobile phone manufacturing, medical equipment manufacturing and network data transmission and other aspects, will greatly promote the development of all walks of life.

At the same time, in order to meet the needs of all walks of life, many colleges and universities have set up the embedded system related courses. However, the teaching mode of embedded system course is obsolete, and its practical application is poor. It has become one of the major difficulties in the teaching. On the basis of switching to English teaching, some experimental teaching content is transplanted to the theoretical part, which increases the time of experiment teaching. In the experiment teaching, the experiment link is designed with the actual project as the core. In the gradual completion of the project at the same time, practical training of students' practical ability and problem-solving skills.

Embedded system teaching is complex, its complexity is that it involves a wide range of knowledge, theory and practice[2-4]. Students need to learn through theory, specific design, hands-on experiments and other processes in order to master the embedded system related knowledge and technology. Aiming at the theoretical and experimental teaching methods, the traditional teaching method is not applicable to embedded systems teaching and has brought the embedded teaching into the wrong zone. Therefore, it is extremely important to carry out exploratory teaching reform for embedded courses. It is the primary duty for all colleges and universities to cultivate high-quality engineering personnel to meet the needs of the society[5].

Moreover, learning embedded systems requires extensive knowledge and familiarity with operating skills, and the students can master the course more effectively through practice on the basis of mastering certain theoretical knowledge. Therefore, we can further our consolidate and knowledge by solving practical problems in practice. 


\section{Problems in Experimental Teaching of the Embedded System}

\subsection{The content of experiment is too monotonous}

Nowadays, the embedded experiment teaching is mostly confirmatory experiment, and there is no correlation between each experiments. A single experiment can enhance students' understanding of embedded basic knowledge, but the whole system cannot be connected in series which cannot meet the requirement of embedded engineers in the society. This teaching mode can not cultivate the creativity of divergent thinking.

\subsection{Students are overly dependent on the teacher}

The education of colleges and universities leads to the fact that today's students are overly dependent on teachers. Most students will repeat the teacher's operation, only recording and sorting all the experimental data. Once they are in trouble, most students are at a loss.

\subsection{Laboratory equipment outdated, lack of good experimental discussion environment}

Due to various reasons, university laboratory equipment cannot be replaced for many years, lack of timely repair of damaged equipment. Because the laboratory research is out of touch with social needs, so it cannot be adapted to the development of social technology.

\section{Embedded System Course}

Based on the theory teaching system, this paper presents a two-levels experimental design, from simple to complex, which can meet the experimental requirements at different stages. The main purpose of the basic experiment is to expand the thinking. Aiming at the specific topic, students need to abstract the main functions according to the requirements of the topic, and then realize the project requirements in theory. This method emphasizes on the theoretical hardware/software co-design by simulation. Using this method, two levels can be carried out step by step, combining the actual project with embedded design theory.

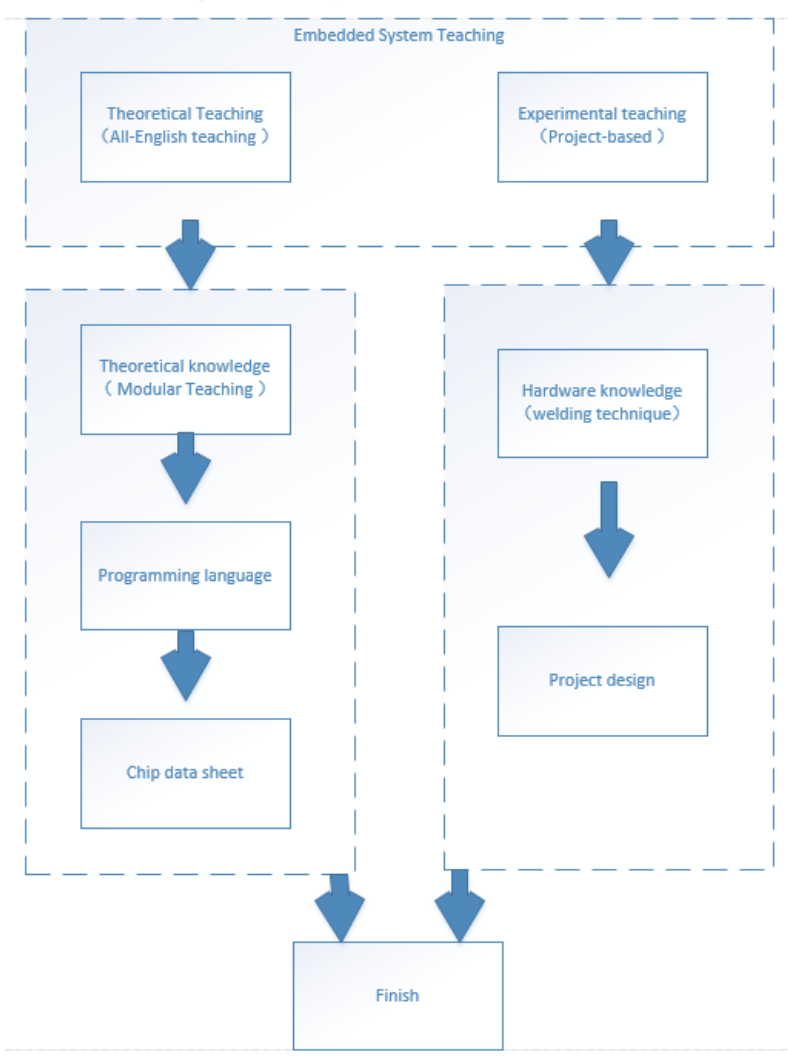

Fig.1. The embedded system course

The traditional experimental teaching is mainly based on simple programming language learning and verification simulation. It has not done add-on functions' development of embedded system 
equipment, and has not been combined with actual development of the project. Using embedded experimental teaching in English, i.e., the description of task requirements in English, the maximum close to the syntax and programming operation, the design will focus on the design and synthesis of hardware system, which greatly improves the overall architecture of the embedded design, completely different from other programming teaching. The teaching contents revolve around basic knowledge learning, programming language learning and chip learning of embedded system. The purpose of teaching is to make use of the advantages of English teaching to broaden the content of theoretical teaching, and to better connect and expand experimental teaching. Basic embedded system structure teaching is not more than half of the total class hour, then according to the project selection, led by the teacher, grouping learning will be completed in remaining time. In the new curriculum system of the embedded system, the whole system can put language learning and hardware chip learning into classroom learning, the content can be increased greatly, combining embedded system teaching with practical application.

\section{Case analysis: design of temperature acquisition system}

\subsection{Embedded system}

An embedded system device is usually made up of an embedded computer system and an executive device, and the Embedded computer system is the core of the whole embedded system. It consists of hardware layer, middle layer, system software layer and application software layer. The hardware layer contains embedded microprocessor, memory (SDRAM, ROM, Flash, etc.), general device interface and I/O interface (A/D, D/A, I/O, etc.). On the basis of an embedded processor, a power supply circuit, a clock circuit and a memory circuit are added to form an embedded core control module. Among them, the operating system and applications can be solidified in ROM. There is an intermediate layer between the hardware layer and the software layer, also known as the hardware abstraction layer (Hardware, Abstract, Layer, HAL) or the board support package (Board, Support, Package, BSP) that separates the system upper layer software (Applications) from the underlying hardware, enabling the bottom of the system. The layer driver has nothing to do with the upper layer, and the upper software developer does not have to care about the underlying hardware, depending on the interface provided by the BSP layer Develop. This layer generally contains initialization of underlying hardware, data input/output operations, and hardware device configuration. The system software layer is composed of real-time multitask operating system (Real-time, Operation, System, RTOS), file system, graphical user interface (Graphic, User, Interface, GUI), network system and general component module.

\subsection{Experimental curriculum arrangement}

The majority of colleges and universities only have single chip microcomputer teaching, the development of embedded systems directly on the Linux system will cause most students to shrink, in the first experimental course embedded in the environment of windows ADS1.2 ARM system development program, after gradually mastering the control of the peripheral devices of the processor, the uboot transplantation, operation system transplantation, file system transplantation, GUI porting and driver development.

All the students are divided into three groups in the experimental class, each group completed the design of temperature monitoring system respectively. Students already have a good understanding of UART, ADC, NAND, FLASH, LED, LCD, timing interrupt module by the basic experimental course. Therefore, the purpose of the experimental course is to enable students to use their existing knowledge of the functional design to realize this system.

\subsection{Realization of temperature monitoring system}

In the experimental class, we used the S3C2440 module, the Linux operating system and the DS18B20 temperature sensor to design the temperature detection system. In the system, we use S3C2440's common IO port GPIO0 to hang on a number of DS18B20 sensors to complete the 
precise control of the timing of the temperature sensor; The schematic diagram of the system hardware is shown in Fig. 2.

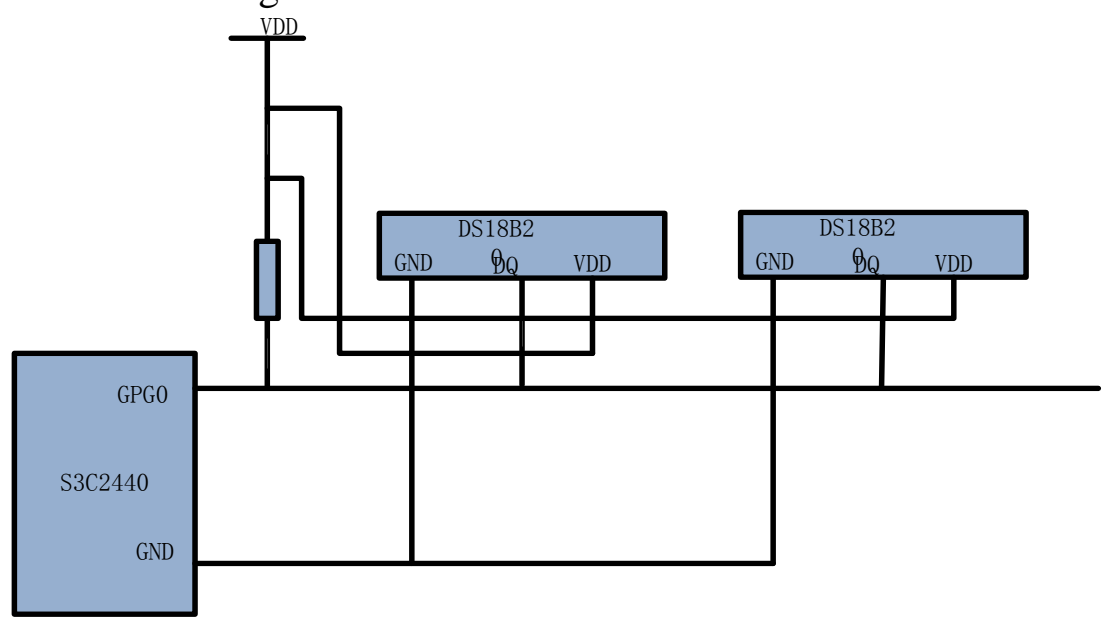

Fig.2. Temperature acquisition system

In order to use DS18B20 sensors, the control of DS18B20 must be strictly conducted in accordance with its logical sequence diagram firstly; Respectively, to complete the DS18B20 read (write), a function and reset the sub function of the preparation, See the DS18B20 manual for the timing chart, and Use the important data structure struct file_operations to implement the linux driver function.

\subsection{Experimental result}

This case uses S3C2440 as a micro-controller, and realizes the temperature detection system based on ARM-linux system. The utility model has the advantages of high data processing capacity and Reduce the cost of the host computer.

The project teaching method is a kind of live ammunition exercises and can cultivate students' ability to analyze and solve problems, fully explore the creative potential of students. It not only embodies the leading role of the teacher, but also plays the main role of the students, which can better reflect the progress of higher education.

\section{Conclusion}

The experimental teaching of embedded system requires very high theoretical and practical ability for students. This courses not only requires students to master a variety of programming languages (C language, HDL language, etc.), the principles of computer composition, operating systems, etc., but also requires them to train the spirit of exploration, problem-solving ability and practical ability. Through the teaching reform, we have preliminarily explored the micro mechanism of the theoretical teaching and practical teaching of the basic project. However, it also puts forward higher requirements for teachers. For example, teachers need a high level of English and a wide range of teaching coverage, not only skilled welding technology but also the project development. From the experimental results, this teaching model has improved the students' understanding of embedded systems, and students have learned to apply theoretical knowledge to practice. Compared with the traditional computer teaching, experiment teaching based on system design can improve the enthusiasm of students'.

\section{Acknowledgement}

In this paper, the research was sponsored by 2017 annual key curriculum construction project of Municipal Education Commission (Principles of Computer Composition) and Construction of demonstrative courses for foreign students in Shanghai in 2015 (Embedded System Design and Application). 
Thanks to computer science and technology hardware course group teaching team of Shanghai Ocean University.

\section{References}

[1] Xian Jin, Jia Deliang, Bi Sheng. Preliminary research on the teaching reform of the experimental course of embedded system [J]. laboratory research and exploration, 2011 (8): 295-297+301.

[2] Song Yanyi, Chen Li, Yan Fang. Experimental teaching reform and experimental teaching device development of embedded system. Research and exploration of [J]. laboratory, 2011, v.30, No.184 (7): 252-254.

[3] Chen Kai, Wei Wenbo, Deng Ming. Development of embedded system teaching experiment board [J]. experimental technology and management, 2016 (1): 82-85.

[4] Luo Yigui. Experimental teaching practice of embedded system based on problem solving. Research and exploration of [J]. laboratory, 2012, v.31, No.200 (10): 389-391.

[5] Liu Jing. Design and development of embedded teaching experiment platform based on ARM. [D]. University of Electronic Science and technology of China, 2014.

[6] Chen Xi. Research and practice of embedded remote experiment system [D]. Beijing University of Posts and Telecommunications, 2014.

[7] Huang Xiaoling, Duan Wei, Zhao Jianke. Exploration and practice of experimental teaching system of embedded system [J]. experimental technology and management, 2006, 23 (4): 85-87.

[8] Zhang Lilian. Study on multi temperature monitoring system of greenhouse based on [J]. S3C2440 Anhui agricultural science, 2010, 38 (20): 10923-10924. 